\title{
The Impact of COVID-19 on US Radiation Oncology Residents
}

\author{
Samuel Kosydar ${ }^{1} \cdot$ Michael W. Woodfin ${ }^{1} \cdot$ Lia M. Halasz $^{1,2} \cdot$ Smith Apisarnthanarax ${ }^{1,2} \cdot$ Ramesh Rengan $^{1,2}$. \\ Simon S. Lo ${ }^{1,2}$
}

Accepted: 7 March 2021 / Published online: 11 March 2021

(C) American Association for Cancer Education 2021

\begin{abstract}
The purpose of our study is to assess the impact of COVID-19 on the clinical responsibilities, training, and wellness of US radiation oncology residents. An anonymous cross-sectional survey was sent to all 91 radiation oncology residency programs in the USA. The survey included questions related to demographics, changes in clinical duties and training, job prospects, and wellness indicators. Univariate and multivariate logistic regression analyses were used to evaluate factors associated with residents endorsing high satisfaction with their departments' response to COVID-19. A total of 96 residents completed the survey from 67 US radiation oncology programs. In the multivariate logistic regression model, remote contouring (OR: 3.91 (95\% CI: $1.11,13.80), p=0.03$ ) and belief that one will be adequately trained to independently practice after completing residency (OR: $4.68(1.12,19.47), p=0.03)$ were significantly associated with high resident satisfaction with their department's response to COVID-19. Most residents indicated that hypofractionation was encouraged to a greater extent $(n=79,82.3 \%)$, patients were triaged by disease risk $(n=67,69.8 \%)$, and most agreed/strongly agreed that they have been provided with adequate personal protective equipment (PPE) $(n=85,88.5 \%)$. The COVID-19 pandemic has affected the training and wellness of radiation oncology residents. Our analysis suggests that radiation oncology programs might increase resident satisfaction with their department's response to COVID-19 by enabling remote contouring and working with residents to identity and remedy possible concerns regarding their ability to independently practice post residency.
\end{abstract}

Keywords COVID-19 $\cdot$ Radiation oncology $\cdot$ Radiation oncology residents

\section{Introduction}

COVID-19 has significantly affected the practice of medicine and impacted clinical training worldwide. While prior work has examined the effect of COVID-19 on the training of residents in otolaryngology [6], urology [10], dermatology [13], and ophthalmology [21] in addition to young arthroplasty surgeons [7], no such work, to our knowledge, has studied how COVID-19 has impacted radiation oncology residents. Factors such as increased vulnerability to respiratory infection in patients with cancer and the multiplicity of appointments per fractionation schedule may pose particular challenges for radiation oncology residents in combating the COVID-19 pandemic [17]. The American Society of Radiation

Simon S. Lo

simonslo@uw.edu

School of Medicine, University of Washington, Seattle, WA, USA

2 Department of Radiation Oncology, University of Washington, 1959 NE Pacific St, Seattle, WA 98195, USA
Oncology (ASTRO) has produced a collection of guidelines and resources [4], including the Johns Hopkins University Program in a Pandemic, which indicated that its residents may be called upon to serve institutional needs and that they should limit their clinic exposure to their primary site [8]. Mohammadi and colleagues assessed the policies of four radiation oncology residency programs in Florida and found that all four developed triage protocols to prioritize patients based on disease risk, encouraged hypofractionation, restricted events, and took steps to promote wellness among their residents [14]. To better understand how COVID-19 has affected the responsibilities, training, and wellness of radiation oncology residents, we surveyed all accredited US programs.

\section{Methods and Materials}

An anonymous cross-sectional survey was developed based on prior published radiation oncology residency surveys [3, 16], COVID-19 related residency surveys [6, 10], and faculty input. Residents were asked to self-report data and 
participation was entirely voluntary. Anxiety, depression, and burnout were assessed using the GAD-2 [12], PHQ-2 [11], and MBI-2 [20], respectively. All 91 US radiation oncology residency programs accredited through the Accreditation Council for Graduate Medical Education (ACGME) [2] were emailed the survey. The survey was conducted using Catalyst WebQ software and approved by the Institutional Review Board (IRB)/Human Subjects Division at the University of Washington. Univariate logistic regression was conducted to better understand the factors that may affect high satisfaction with a department's response to COVID-19 (scoring 4 or 5 on the question: How satisfied are you with your department's response to COVID-19? 1- least satisfied, 5- most satisfied). Results of the univariate regression that yielded $p<0.1$ were subsequently included in multivariate logistic regression analysis [16]. $p$ value $<0.05$ was considered significant. Analyses were conducted with SPSS Statistics Version 27.

\section{Results}

Ultimately, 96 residents completed the survey, representing $12.5 \%$ of all US radiation oncology residents $(n=771)$ per academic year 2019-2020 data. Among the 67 US residency programs that confirmed they would forward the survey to their residents, the response rate was $16.3 \%$. Over a third of respondents were female $(n=34,35.4 \%)$ and one respondent indicated that sex was not male or female $(n=1,1.0 \%)$. Most respondents were married $(n=52,54.2 \%)$ and more than a quarter reported having children $(n=27,28.1 \%)$. Less than $10 \%$ lived in households with anyone over the age of 65 or with comorbid conditions ( $n=7,7.3 \%)$. Additional demographic information is available in Online Resource 1.

Table 1 describes the impact of COVID-19 on the clinical practice and training of radiation oncology residents. More than half of respondents had been in contact with COVID19 positive patients ( $n=53,55.2 \%)$ and many were concerned about infecting a family member, loved one, or close friend (scores $4-5, n=50,52.1 \%$ ). Overall, most respondents agreed/strongly agreed with the statement that they have been provided with adequate PPE $(n=85,88.5 \%)$ and were very satisfied with their department's response to COVID-19 (scores 4-5, $n=69,71.9 \%$ ). Overwhelmingly, respondents reported that hypofractionation was encouraged to a greater extent $(n=79,82.3 \%)$, contouring was being done remotely ( $n=74,77.1 \%$ ), and that patients were triaged by disease risk $(n=67,69.8 \%$ ). More than two thirds of respondents indicated that during the COVID-19 pandemic they were seeing fewer patients than before $(n=65,67.7 \%)$, and more than half did not experience a change to the number of patients they saw while on call $(n=54,56.3 \%)$. Less than a quarter of respondents $(n=21,21.9 \%)$ endorsed working $\leq 50 \mathrm{~h}$ per week before the pandemic, whereas more than a third reported working $\leq 50 \mathrm{~h}$ during the pandemic $(n=34,35.4 \%)$. Although most respondents indicated there was generally no change in training on brachytherapy, unsealed sources, intracranial SRS, or SBRT, a third of respondents $(n=32,33.3 \%)$ reported their training on brachytherapy decreased. No respondent endorsed an increase in training on either brachytherapy or unsealed sources. In comparison, 16 (16.7\%) respondents noted an increase in SBRT training.

Table 1 also summarizes respondents' beliefs regarding how COVID-19 may affect their future training and career prospects and describes the impact of COVID-19 on the wellness of respondents. Approximately half of the respondents agreed/strongly agreed with the statement that COVID-19 will negatively impact their ability to find a job $(n=49,51.0 \%)$. Overall, most respondents also agreed or strongly agreed $(n=82,85.4 \%)$ that they would be adequately trained to independently practice radiation oncology after completing residency and, if given the opportunity, would choose a residency in radiation oncology again $(n=75,78.1 \%)$. More than half of responding residents did not report changes to time spent sleeping $(n=58,60.4 \%)$, overall level of depression $(n=68,70.8 \%)$, or burnout $(n=59$, $61.5 \%$ ), but did endorse increased overall level of anxiety $(n=56,58.3 \%)$. Almost half of respondents $(n=$ $44,45.8 \%)$ noted decreased time spent with family and no change in time spent on research $(n=46,47.9 \%)$. Most respondents screened negative for depression using the PHQ-2 ( $n=84,87.5 \%)$, negative for anxiety using the GAD-2 ( $n=80,83.3 \%)$, and negative for burnout using the MBI-2 ( $n=76,79.2 \%)$.

Logistic regression was conducted to better understand factors associated with residents' satisfaction with their department's response to COVID-19. For univariate logistic regression (Table 2), high department satisfaction (scores 4-5) was associated with performing contouring remotely (OR: 5.71, 95\% CI $(2.02,16.20), p=0.001)$, adequate access to PPE, defined as agree/strongly agree (OR: $5.69(1.51,21.44), p=$ 0.01 ), and agreeing/strongly agreeing with the statement "I would choose radiation oncology again" (OR: 3.41 (1.22, 9.56), $p=0.02$ ). It was negatively associated with working more than $70 \mathrm{~h}$ per week during the COVID-19 pandemic (OR: $0.13(0.02,0.74), p=0.02)$, decreased time spent with family (OR: $0.39(0.15,0.97), p=0.04)$, a positive screen for depression on the PHQ-2 (OR: $0.18(0.05,0.67), p=0.01$ ), and a positive screen for anxiety on the GAD-2 (OR: 0.27 $(0.09,0.85), p=0.03)$. For multivariate logistic regression (Table 3 ), high department satisfaction was significantly associated with remote contouring (OR: 3.91 (1.11, 13.80), $p=$ 0.03 ) and agreement/strong agreement with the statement "I feel that I will be adequately trained to independently practice radiation oncology after completing residency" (OR: 4.68 $(1.12,19.47), p=0.03)$. 
Table 1 Survey responses related to impact of COVID-19 on clinical practice, training, career prospects, and wellness

Have you been in contact with COVID-19 positive patients?

Owing to COVID-19, did you have duties outside of your standard responsibilities?

Was hypofractionated treatment encouraged to a greater extent?

Is/was contouring being done remotely?

Regarding patient prioritization, were patients triaged by disease risk?

COVID-19 impact on the number of cases you had during a normal week?

How did COVID-19 impact the number of patients you saw on call?

Training with brachytherapy

Training with unsealed sources

Training with intracranial SRS

Training with SBRT

Time spent sleeping

Time spent on research

Time spent with family

My overall level of anxiety

My overall level of burnout

My overall level of depression

How concerned are you about contracting COVID-19? (1 is low, 5 is high)

How concerned are you that you may infect a family member, loved one, close friend with COVID-19? (1 is low, 5 is high)

How satisfied are you with your department's response to COVID-19?

(1 least satisfied, 5 most satisfied)

I have been provided adequate PPE.

Strongly disagree

Disagree

Neutral

Agree

Strongly agree

Before the COVID-19 pandemic, in an average week, I spent

$<40 \mathrm{~h}$

$41-50 \mathrm{~h}$

$51-60 \mathrm{~h}$

$61-70 \mathrm{~h}$

$>70 \mathrm{~h}$

During the COVID-19 pandemic, in an average week, I spent

$<40 \mathrm{~h}$

41-50 h

51-60 h

61-70 h

$>70 \mathrm{~h}$

COVID-19 will negatively impact my ability to find a job.

Strongly disagree

Disagree

Neutral

Agree

Strongly agree

\begin{tabular}{|c|c|c|}
\hline Yes $N(\%)$ & No $N(\%)$ & Unsure $N(\%)$ \\
\hline $53(55.2 \%)$ & $28(29.2 \%)$ & $15(15.6 \%)$ \\
\hline $14(14.6 \%)$ & $68(70.8 \%)$ & $\begin{array}{l}14(14.6 \%) \text { (no but there } \\
\text { are plans in place) }\end{array}$ \\
\hline $79(82.3 \%)$ & $9(9.4 \%)$ & $8(8.3 \%)$ \\
\hline $74(77.1 \%)$ & $20(20.8 \%)$ & $1(1.0 \%)$ \\
\hline $67(69.8 \%)$ & $12(12.5 \%)$ & $17(17.7 \%)$ \\
\hline Increased $N(\%)$ & Decreased $N(\%)$ & No change $N(\%)$ \\
\hline $2(2.1 \%)$ & $65(67.7 \%)$ & $29(30.2 \%)$ \\
\hline $3(3.1 \%)$ & $39(40.6 \%)$ & $54(56.3 \%)$ \\
\hline 0 & $32(33.3 \%)$ & $60(62.5 \%)$ \\
\hline 0 & $23(24.0 \%)$ & $69(71.9 \%)$ \\
\hline $3(3.1 \%)$ & $15(15.6 \%)$ & $72(75.0 \%)$ \\
\hline $16(16.7 \%)$ & $10(10.4 \%)$ & $65(67.7 \%)$ \\
\hline $22(22.9 \%)$ & $15(15.6 \%)$ & $58(60.4 \%)$ \\
\hline $34(35.4 \%)$ & $15(15.6 \%)$ & $46(47.9 \%)$ \\
\hline $25(26.0 \%)$ & $44(45.8 \%)$ & $26(27.1 \%)$ \\
\hline $56(58.3 \%)$ & $2(2.1 \%)$ & $37(38.5 \%)$ \\
\hline $29(30.2 \%)$ & $7(7.3 \%)$ & $59(61.5 \%)$ \\
\hline $24(25.0 \%)$ & $3(3.1 \%)$ & $68(70.8 \%)$ \\
\hline Median & $N($ scores $1-3)$ & $N($ scores 4-5) \\
\hline 3 & $78(81.3 \%)$ & $18(18.8 \%)$ \\
\hline 3 & $46(47.9 \%)$ & $50(52.1 \%)$ \\
\hline 4 & $27(28.1)$ & $69(71.9 \%)$ \\
\hline
\end{tabular}

0

$3(3.1 \%)$

$8(8.3 \%)$

$42(43.8 \%)$

$43(44.8 \%)$

at work.

$2(2.1 \%)$

$19(19.8 \%)$

$39(40.6 \%)$

25 (26.0\%)

$9(9.4 \%)$

at work.

$4(4.2 \%)$

$30(31.3 \%)$

$33(34.4 \%)$

$21(21.9 \%)$

$7(7.3 \%)$

$4(4.2 \%)$

$11(11.5 \%)$

$32(33.3 \%)$

$32(33.3 \%)$

$17(17.7 \%)$ 
Table 1 (continued)

I feel that I will be adequately trained to independently practice radiation oncology after completing residency.

Strongly disagree

Disagree

0

Neutral

$2(2.1 \%)$

Agree

Strongly agree

I would choose a residency in radiation oncology again.

Strongly disagree

Disagree

$12(12.50 \%)$

$40(41.7 \%)$

$42(43.8 \%)$

Neutral

$3(3.1 \%)$

Agree

Strongly agree

$11(11.5 \%)$

$29(30.2 \%)$

PHQ-2 for depression

$46(47.9 \%)$

GAD-2 for anxiety

Positive screen $N(\%)$

$11(11.5 \%)$

$15(15.6 \%)$

$19(19.8 \%)$

\section{Discussion}

This is the first study, to our knowledge, that assesses the impact of the COVID-19 pandemic on the clinical duties, training, and wellness of radiation oncology residents. Our multivariate analysis showed that remote contouring and endorsement of the belief that one will be adequately trained to independently practice after residency was significantly associated with high resident satisfaction with their department's response to COVID-19. One possibility is that residents who were already satisfied with their department were more likely to be satisfied with their department's COVID-19
Table 2 Univariate logistic regression, "high satisfaction with department response to COVID19 " as endpoint

\begin{tabular}{|c|c|c|c|}
\hline & Reference & Odds ratio $(95 \% \mathrm{CI})$ & $p$ value \\
\hline Age & a & $1.02(0.86,1.21)$ & 0.81 \\
\hline Sex & Female & $0.59(0.24,1.46)$ & 0.25 \\
\hline Year of training & PGY-5 & $0.76(0.29,1.98)$ & 0.58 \\
\hline Contact with COVID-19 positive patients & Yes & $0.64(0.26,1.60)$ & 0.34 \\
\hline $\begin{array}{l}\text { Clinical duties outside of your standard } \\
\text { responsibilities }\end{array}$ & Yes & $0.46(0.14,1.48)$ & 0.19 \\
\hline Hypofractionation treatment encouraged & Yes & $1.51(0.50,4.59)$ & 0.47 \\
\hline Contouring done remotely & Yes & $5.71(2.02,16.20)$ & 0.001 \\
\hline Patients triaged by disease risk & Yes & $0.43(0.14,1.27)$ & 0.13 \\
\hline Change in number of cases per week & Fewer cases & $1.68(0.67,4.26)$ & 0.27 \\
\hline Change in number patients seen on call & Fewer & $0.80(0.33,1.98)$ & 0.63 \\
\hline Concern about contracting COVID-19 & Score $4 / 5$ & $0.74(0.25,2.21)$ & 0.59 \\
\hline $\begin{array}{l}\text { Concern about infecting family members } \\
\text { and friends }\end{array}$ & Score $4 / 5$ & $0.67(0.27,1.65)$ & 0.38 \\
\hline Adequate PPE & Agree/strongly agree & $5.69(1.51,21.44)$ & 0.01 \\
\hline Work hours during COVID-19 & $>70 \mathrm{~h}$ & $0.13(0.02,0.74)$ & 0.02 \\
\hline $\begin{array}{l}\text { COVID-19 will negatively impact } \\
\text { ability to find job }\end{array}$ & Agree/strongly agree & $0.51(0.20,1.27)$ & 0.15 \\
\hline $\begin{array}{l}\text { Adequately trained to independently practice } \\
\text { after residency }\end{array}$ & Agree/strongly agree & $3.1(0.97,9.91)$ & 0.06 \\
\hline Would choose radiation oncology again & Agree/strongly agree & $3.41(1.22,9.56)$ & 0.02 \\
\hline Time spent sleeping & Increased & $2.07(0.63,6.81)$ & 0.23 \\
\hline Time spent on research & Increased & $2.45(0.88,6.85)$ & 0.09 \\
\hline Time spent with family & Decreased & $0.39(0.15,0.97)$ & 0.04 \\
\hline Depression & Positive screen & $0.18(0.05,0.67)$ & 0.01 \\
\hline Anxiety & Positive screen & $0.27(0.09,0.85)$ & 0.03 \\
\hline Burnout & Positive screen & $0.61(0.21,1.77)$ & 0.37 \\
\hline
\end{tabular}

${ }^{\text {a }}$ Reference is for every 1 year increase in age 
Table 3 Multivariate logistic regression, "high satisfaction with department response to COVID19 " as endpoint

\begin{tabular}{llll}
\hline & Reference & Odds ratio $(95 \%$ CI $)$ & $p$ value \\
\hline Contouring done remotely & Yes & $\mathbf{3 . 9 1}(\mathbf{1 . 1 1}, \mathbf{1 3 . 8 0})$ & $\mathbf{0 . 0 3}$ \\
Adequate PPE & Agree/strongly agree & $3.77(0.70,20.18)$ & 0.12 \\
Work hours during COVID-19 & $>70 \mathrm{~h}$ & $0.19(0.03,1.42)$ & 0.11 \\
Adequately trained to independently & Agree/strongly agree & $\mathbf{4 . 6 8}(\mathbf{1 . 1 2}, \mathbf{1 9 . 4 7})$ & $\mathbf{0 . 0 3}$ \\
$\quad$ practice after completing residency & & $1.48(0.36,6.05)$ & 0.59 \\
Would choose radiation oncology again & Agree/strongly agree & $1.92(0.49,7.48)$ & 0.35 \\
Time spent on research & Increased & $0.62(0.19,2.06)$ & 0.44 \\
Time spent with family & Decreased & $0.45(0.03,6.03)$ & 0.54 \\
Depression & Positive screen & $1.37(0.17,10.88)$ & 0.77 \\
Anxiety & Positive screen &
\end{tabular}

response. Another possibility is that departments that are particularly good at training residents, and endorsed by their residents as such, excelled at adapting to COVID-19. Regarding contouring, one possible interpretation of this finding is that residents greatly valued a department's efforts to reduce COVID-19 exposure risk by reducing in-person duties which can be done, within reason, away from clinic. Contouring remotely necessarily requires the ability to externally access institutional servers, a process which may be challenged by security concerns and logistical issues such as adequate internet connection. Although there is little published literature regarding remote contouring [15], a versatile approach that can withstand the uncertainty of COVID-19 and meet the needs of residents, while ensuring high quality work, may be a prudent departmental goal.

Our survey results are reflective of published radiation oncology institutional reports. In congruence with Mohammadi et al. [14], who examined the COVID-19 protocols of four residency programs in Florida, as well as Tey et al. [19] in Singapore, and Buckstein et al. [5] in New York, we generally found that hypofractionation was encouraged and that patients were triaged by disease risk (Table 1). Simcock and colleagues cautioned that brachytherapy delivery may be particularly challenging in the COVID-19 environment and that one should plan for alternatives using external beam therapy [18]. This expert opinion was validated in our survey that found residents reporting that brachytherapy training demonstrated the most significant decline ( $n=32,33.33 \%)$ compared to other treatment modalities. Promoting resident wellbeing during the COVID-19 pandemic has been a priority of numerous radiation oncology programs $[5,9,14]$. Our results indicate that most responding radiation oncology residents screened negative for clinical depression, anxiety, and burnout and that most endorsed no change in their overall level of depression or burnout since COVID-19 began, however more than half indicated an increase in their overall level of anxiety $(n=56,58.3 \%)$.

Our survey results suggest that the experience of radiation oncology residents during the COVID-19 pandemic has been similar to peers in other fields. In a survey of ENT residents,
$79 \%$ were moderately to extremely worried about transmitting COVID-19 to family and friends [6], similar to our reported $81.3 \%$ (scores $3-5, n=78$ ) for radiation oncology residents. While a survey of urology residents indicated that $22 \%(n=$ 72) were redeployed to outside duties [10], our redeployment rate was $14.6 \%(n=14)$. In addition, urology residents' adequate access to PPE was reported as a mean score of 3.4 (scales $1-5$, where 1 is strongly disagree and 5 is strongly agree) [10], in contrast $88.5 \%(n=85)$ of responding radiation oncology residents agreed/strongly agreed that they have been provided with adequate PPE. Resident satisfaction with department response to COVID-19 was $73 \%$ in ENT [6] while $71.9 \%(n=69)$ of radiation oncology residents were very satisfied (scores 4-5).

Our field wide response rate of $12.5 \%(n=96)$ is consistent with numerous published surveys in other fields assessing the impact of COVID-19 on residents. Cai et al. totaled 82 responses from 51 otolaryngology programs [6], which according to the ACGME 2019-2020 data ( $n=1689$ otolaryngology residents) [1] is a $4.9 \%$ field wide response rate. Li et al. garnered 140 dermatology residents from 50 residency programs [13], representing $8.8 \%$ of the entire field $(n=1594)$ per ACGME data [1]. Culp et al. queried members of the Young Arthroplasty Group (orthopedic residents, fellows, and surgeons within 5 years of practice) with a total of 157 responses, including 39 residents [7]. Per ACGME 2019-2020 data [1], there were 4342 orthopedic residents, so this survey represented less than $0.90 \%$ of the entire field of residents. Woodfin et al. surveyed 234 ophthalmology residents from 57 institutions, with a field wide response rate of $15.5 \%$ [21]. Khusid et al.'s survey of urology residents [10] yielded a field wide response rate of 20.5\% using ACGME data 2019-2020 [1].

\section{Limitations}

Our analysis is subject to several limitations. First, we did not collect geographic location such as state, which certainly may have provided useful information since the impact of COVID19 was not uniform across the country. However, considering 
the size of radiation oncology programs, with some states having only a single program if at all, and the personal nature of some questions being asked, we wanted to provide the greatest level of anonymity possible for residents. Although we reported the total of number of departments that agreed to forward the survey to their residents, we did not collect data tying individual responses to particular departments, so as to encourage department participation by providing anonymity. Thus, we are unable to comment on department characteristics, such as percentage of departments whose residents reported adequate access to PPE. Additionally, owing to our sample size, small but consistent with other published residency surveys during COVID-19, and possible responder bias, which is inherent to any voluntary survey, our results may not necessarily reflect the entire field.

\section{Conclusion}

Most responding radiation oncology residents had contact with COVID-19 positive patients and were concerned about infecting family members or loved ones. Many reported increased overall anxiety during the pandemic. However, they also indicated adequate access to PPE and were generally satisfied with their departments' COVID-19 response. Hypofractionation was encouraged to a greater extent and patients were generally triaged by disease risk. Regarding training with treatment modalities, brachytherapy saw the biggest decline. In addition, our analysis indicated remote contouring was strongly associated with high resident satisfaction with their department's COVID-19 response, which may further support implementation of remote contouring in programs that have yet to do so.

Supplementary Information The online version contains supplementary material available at https://doi.org/10.1007/s13187-021-01993-5.

Code Availability Not applicable.

Data Availability In order to protect the privacy of survey respondents, additional data is not available.

\section{Declarations}

Conflict of Interest Dr. Lo is a member of the Gamma Knife ICON Expert Group, Elekta AB, outside the submitted work. All other authors declare no conflicts of interest.

\section{References}

1. Accreditation Council for Graduate Medical Education. ACGME data retrieval system. https://apps.acgme-i.org/ads/Public/Request/ PublicDataRequest. Accessed 18 October 2020
2. Accreditation Council for Graduate Medical Education. List of programs by specialty. https://apps.acgme-i.org/ads/Public/Reports/ Report/1. Accessed 16 July 2020

3. Ahmed AA, Ramey SJ, Dean MK, Yoo SK, Holliday E, Deville C, Takita C, Vapiwala N, Wilson LD, Jagsi R, Thomas CR Jr, Yechieli R (2018) Resident satisfaction with radiation oncology training. Adv Radiat Oncol 3(3):234-239. https://doi.org/10.1016/j.adro. 2018.03.003

4. American Society for Radiation Oncology. COVID-19 clinical guidance https://www.astro.org/Daily-Practice/COVID-19Recommendations-and-Information/Clinical-Guidance. Accessed 13 July 2020

5. Buckstein M, Skubish S, Smith K, Braccia I, Green S, Rosenzweig K (2020) Experiencing the Surge: report from a large New York radiation oncology department during the COVID-19 pandemic. Adv Radiat Oncol 5(4):610-616. https://doi.org/10.1016/j.adro. 2020.04.014

6. Cai Y, Jiam NT, Wai KC, Shuman EA, Roland LT, Chang JL (2020) Otolaryngology resident practices and perceptions in the initial phase of the U.S. COVID-19 pandemic. Laryngoscope. https://doi.org/10.1002/lary.28733

7. Culp BM, Frisch NB (2020) COVID-19 impact on young arthroplasty surgeons. J Arthroplast 35:S42-S44. https://doi.org/ 10.1016/j.arth.2020.04.058

8. Department of Radiation Oncology \& Molecular Radiation Sciences Johns Hopkins University. 6 April 2020. An integrated program in a pandemic. https://www.astro.org/ASTRO/media/ $\mathrm{A} \mathrm{S} \mathrm{T} \mathrm{R} \mathrm{O} \mathrm{/} \mathrm{D} \mathrm{a} \mathrm{i} 1$ y $\% 20 \mathrm{Practice} / \mathrm{P} \mathrm{DFs} /$ JHUPrograminaPandemic040320.pdf. Accessed 13 July 2020

9. Dinh TT, Halasz LM, Ford E, Rengan R (2020) Radiation therapy in King County, Washington during the COVID-19 pandemic: balancing patient care, transmission mitigation, and resident training. Adv Radiat Oncol 5(4):544-547. https://doi.org/10.1016/j. adro.2020.03.007

10. Khusid JA, Weinstein CS, Becerra AZ, Kashani M, Robins DJ, Fink LE, Smith MT, Weiss JP (2020) Well-being and education of urology residents during the COVID-19 pandemic: results of an American national survey. Int J Clin Pract 74(9):e13559. https:// doi.org/10.1111/ijcp.13559

11. Kroenke K, Spitzer RL, Williams JB (2003) The Patient Health Questionnaire-2: validity of a two-item depression screener. Med Care 41(11):1284-1292. https://doi.org/10.1097/01.Mlr. $0000093487.78664 .3 \mathrm{c}$

12. Kroenke K, Spitzer RL, Williams JBW, Monahan PO, Lowe B (2007) Anxiety disorders in primary care: prevalence, impairment, comorbidity, and detection. Ann Intern Med 146(5):317-325. https://doi.org/10.7326/0003-4819-146-5-200703060-00004\% m17339617

13. Li YM, Galimberti F, Abrouk M, Kirsner RS (2020) US dermatology resident responses about the COVID-19 pandemic: results from a nationwide survey. South Med J 113(9):462-465. https://doi.org/ $10.14423 / \mathrm{smj} .000000000001141$

14. Mohammadi H, De Leo AN, Asher D, Malouff TD, Waddle MR, Figura NB, Patel NV, Bates JE, Parekh AD (2020) The Florida radiation oncology resident experience during coronavirus disease 2019: perspectives and recommendations. Adv Radiat Oncol 5: 727-731. https://doi.org/10.1016/j.adro.2020.05.002

15. Price A, Kim H, Henke LE, Knutson NC, Spraker MB, Michalski J, Hugo GD, Robinson CG, Green O (2020) Implementing a novel remote physician treatment coverage practice for adaptive radiation therapy during the coronavirus pandemic. Adv Radiat Oncol 5(4): 737-742. https://doi.org/10.1016/j.adro.2020.05.007

16. Ramey SJ, Ahmed AA, Takita C, Wilson LD, Thomas CR, Yechieli R (2017) burnout evaluation of radiation residents nationwide: results of a survey of United States residents. Int J Radiat 
Oncol Biol Phys 99(3):530-538. https://doi.org/10.1016/j.ijrobp. 2017.06.014

17. Rivera A, Ohri N, Thomas E, Miller R, Knoll MA (2020) The impact of COVID-19 on radiation oncology clinics and patients with cancer in the United States. Adv Radiat Oncol 5:538-543. https://doi.org/10.1016/j.adro.2020.03.006

18. Simcock R, Thomas TV, Estes C, Filippi AR, Katz MS, Pereira IJ, Saeed H (2020) COVID-19: global radiation oncology's targeted response for pandemic preparedness. Clin Transl Radiat Oncol 22: 55-68. https://doi.org/10.1016/j.ctro.2020.03.009

19. Tey J, Ho S, Choo BA, Ho F, Yap SP, Tuan JKL, Leong CN, Cheo T, Sommat K, Wang MLC (2020) Navigating the challenges of the COVID-19 outbreak: perspectives from the radiation oncology service in Singapore. Radiother Oncol 148:189-193. https://doi. org/10.1016/j.radonc.2020.03.030

20. West CP, Dyrbye LN, Sloan JA, Shanafelt TD (2009) Single item measures of emotional exhaustion and depersonalization are useful for assessing burnout in medical professionals. J Gen Intern Med 24(12):1318-1321. https://doi.org/10.1007/s11606-009-1129-z

21. Woodfin M, Bojikian KD, Taravati P, Ding L, Lee MD, Feng S (2020) The Early Impact of COVID-19 on Ophthalmology Resident Training and Wellness. J Acad Ophthalmol 12(02): e292-e297

Publisher's Note Springer Nature remains neutral with regard to jurisdictional claims in published maps and institutional affiliations. 